\title{
Saúde, família e escola: relato de experiência
}

\section{Health, family and school: experience report}

Flávia Emília Cavalcante Valença Fernandes ${ }^{1 *}$, Nadja Maria dos Santosutor ${ }^{1}$, Silvana Coelho de Araújo Anunciação ${ }^{1}$

\begin{abstract}
RESUMO
Compartilhar a experiência relacionada à disciplina eletiva: Saúde, Família e Escola, do Programa de Pósgraduação stricto sensu, Formação de Professores e Práticas Interdisciplinares, da Universidade de Pernambuco campus Petrolina. Saúde e Educação são áreas interdependentes, já que, indivíduos saudáveis aprendem melhor, e uma sociedade com acesso a uma boa educação tem maior probabilidade de ser mais saudável. Metodologia: Trata-se de um estudo descritivo, do tipo relato de experiência. A vivência ocorreu no período de junho a agosto de 2021. Foram 10 encontros semanais no turno matutino. A metodologia de ensino empregada na disciplina compreendeu: encontros assíncronos, a partir do sistema de gerenciamento de conteúdo Google Classroom, e síncronos, neste foi utilizado o serviço de comunicação por vídeo (plataforma Google meet). Considerações finais: A partir dessas experiências entende-se a importante relação existente entre saúde e educação, quais são as políticas públicas voltadas para esses dois campos no Brasil, e as implicações na aprendizagem em ambientes formais e sua relação com o contexto social.
\end{abstract}

Palavras-chave: Aprendizagem; Educação; Promoção da saúde escolar; Saúde.

\begin{abstract}
To share the experience related to the elective discipline: Health, Family and School, from the stricto sensu Graduate Program, Teacher Training and Interdisciplinary Practices, at the University of Pernambuco, campus Petrolina. Health and Education are interdependent areas, as healthy individuals learn better, and a society with access to good education is more likely to be healthier. This is a descriptive study, of the experience report type. The experience took place from June to August 2021. There were 10 weekly meetings in the morning shift. The teaching methodology used in the course included: asynchronous meetings, from the Google Classroom content management system, and synchronous meetings, in which the video communication service (Google meet platform) was used. Based on these experiences, it is possible to understand the important relationship between health and education, which are the public policies aimed at these two fields in Brazil, and the implications for learning in formal environments and its relationship with the social context.
\end{abstract}

Keywords: Learning; Education; Promotion of school health; Health

\section{INTRODUÇÃO}

A saúde e a educação estão inter-relacionadas e interagem entre si e com outros fatores em um contexto social, já que melhorias na educação refletem-se na saúde e vice-

\footnotetext{
${ }^{1}$ Universidade de Pernambuco campus Petrolina ${ }^{1}$.

*E-mail: flavia.fernandes@upe.br
} 
versa (BIRCH, 2019). Diante dessa perspectiva, é importante discussões que envolvam essas duas áreas que são interdependentes, como também, compreender as políticas públicas voltadas para esses setores, e a interferência dessa atuação no processo de aprendizagem em ambientes formais.

É imprescindível estudos que envolvam saúde e educação já que os dois temas estão interligados, de acordo com estudos realizados nas duas áreas, existe um consenso: bons níveis de educação estão relacionados a uma população mais saudável assim como uma população saudável tem maiores possibilidades de apoderar-se de conhecimentos da educação formal e informal (CASEMIRO et al., 2014). Dessa forma, é importante que haja discussão e políticas públicas voltadas para esses dois campos, para o desenvolvimento físico e intelectual de um indivíduo.

A escola tem representado um importante local para o encontro entre saúde e educação abrigando amplas possibilidades de iniciativas tais como: ações de diagnóstico clínico e/ou social estratégias de triagem e/ou encaminhamento aos serviços de saúde especializados ou de atenção básica; atividades de educação em saúde e promoção da saúde (CASEMIRO et al., p. 830).

Lopes et al. (2018), traz que as políticas e programas que atendem a saúde da população são fundamentais para a formação de bons cidadãos, visto que as condições de saúde de uma população influenciam diretamente a aprendizagem. $\mathrm{O}$ ambiente escolar deve oferecer para os educandos pensamentos críticos relacionados à rotina e ao contexto social em que vivem. Além disso é importante alinhar-se com a equipe de saúde no intuito de obter bons índices de desenvolvimento escolar.

É memorável trabalhar temas transversais, como: sexualidade, uso e abuso de substâncias, higiene corporal, nutrição, dentre outras no ambiente escolar. Os profissionais da educação devem estar atentos e disponíveis para desferir ações de promoção da saúde para os educandos, com o objetivo que essas extrapolem as instituições e possam ser inseridas no cotidiano dos mesmos (DOUGLAS, 2021).

Portanto, pretende-se com esse relato de experiência discutir, a partir das experiências obtidas nas aulas da disciplina "Saúde, Família e Escola”, a relação existente entre saúde e educação, envolvendo quais as políticas públicas voltadas para esses dois campos, e quais as implicações na aprendizagem em ambientes formais e sua relação com o contexto familiar. 
O presente trabalho é um relato das experiências vividas durante as aulas do primeiro semestre da disciplina eletiva: Saúde, Família e Educação, do Programa de Pósgraduação Stricto Sensu, Formação de Professores e Práticas Interdisciplinares (PPGFPPI) da Universidade de Pernambuco (UPE) campus Petrolina.

\section{METODOLOGIA}

Trata-se de um estudo descritivo, do tipo relato de experiência. A vivência ocorreu no período de junho a agosto de 2021. Foram 10 encontros, estes aconteciam semanalmente no turno matutino. A metodologia de ensino empregada na disciplina compreendeu: encontros assíncronos, a partir do sistema de gerenciamento de conteúdo Google Classroom, e síncronos neste foi utilizada o serviço de comunicação por vídeo a plataforma Google meet, as atividades foram: aulas teóricas com uso de recursos visuais, rodas de conversas, discussão de artigos científicos, vídeos, palestras com especialistas convidados; e oficinas práticas, que tiveram o objetivo de discutir os aspectos relacionados à saúde no ambiente escolar, de compreender a atuação interdisciplinar entre a saúde e educação, e propor intervenções interdisciplinares voltadas para promoção da saúde e prevenção de agravos à saúde no ambiente escolar.

A disciplina abordou temas voltados para interação entre aspectos relacionados à saúde e a educação no ambiente escolar e a sua relação com o contexto familiar, a percepção dos diversos cenários de influência sobre a saúde em espaços formais e não formais de ensino, e a Educação em Saúde com foco na promoção e prevenção de agravos à saúde pautada na interdisciplinaridade.

Semanalmente, seguindo o cronograma da disciplina era disponibilizado pela docente responsável um tema no Google Classroom, anexo a esse, era disponibilizado artigos, vídeos temáticos e reportagens jornalísticas relacionados ao tema e as respectivas indagações e orientações. De acordo com as orientações dadas nos momentos síncronos e após a leitura, as mestrandas discutiam o tema, realizavam análise crítica dos materiais, ressignificavam a experiência do tema no espaço escolar, elaboravam e compilavam as expectativas e impressões acerca do tema para apresentação na semana seguinte.

Com a finalidade de compartilhar a experiência, serão apresentadas a seguir uma discussão sobre os temas com respectiva fundamentação teórica.

\section{RESULTADOS E DISCUSSÃO}


O PPGFPPI tem como objetivo formar professores (as) nos diferentes níveis de ensino que compreendam a interdisciplinaridade como um desafio prático na pesquisa e nos processos pedagógicos. Entre os objetivos específicos está a de propor atividades interdisciplinares como ferramenta à construção do conhecimento. O programa tem como área de concentração a Educação e é estruturado em duas linhas de pesquisa. A disciplina eletiva desse relato está vinculada a linha de pesquisa Educação, Meio Ambiente e Saúde, essa visa desenvolver estudos interdisciplinares focados na prática docente, com ênfase nos temas transversais definidos pelos Parâmetros Curriculares Nacionais (PCN's) (UPE, 2015).

Durante o estudo dos materiais disponibilizados na disciplina e a partir das temáticas abordadas, percebe-se a importância do trabalho intersetorial entre saúde e educação, ampliando para o contexto familiar, que esses dois campos quando bem trabalhados refletem positivamente, para os aspectos da qualidade de vida, e também promovem uma aprendizagem formal mais significativa, pois a literatura apresenta que, indivíduos mais saudáveis aprendem melhor, e indivíduos com mais conhecimento, são mais saudáveis.

A partir da análise e discussão dos materiais e discussão, as mestrandas elencaram quatro tópicos relacionados a saúde e educação no ambiente escolar.

\section{A interdependência entre Saúde e Educação: contexto histórico}

Durante o período de colonização do Brasil, os habitante desta terra eram, em sua maioria, índios e deportados de Portugal, sendo assim, não houve por parte do colonizador interesse em formar um Estado, consolidar a sociedade e investir em ações de educação e saúde (BARBOSA et al., 2014). Diante dessa perspectiva, entende-se, que questões relacionadas às condições de saúde e qualidade de vida da população, bem como os aspectos relacionados ao acesso da população à educação formal eram negligenciados no tempo do Brasil Colônia.

Ações voltadas para saúde e educação surgiram apenas em 1808, com a vinda da família real Portuguesa para o Brasil, porém, apenas para a elite, fato esse, que gerou a desigualdade social e se prolonga até a atualidade (BARBOSA et al., 2014). O acesso à educação era privilégio apenas para os filhos da elite que iam estudar na Europa.

Após a proclamação da República, que ocorreu em 15 de novembro de 1889, por meio de um golpe militar, a nação torna-se suscetível à influência filosófica positivista 
(BARBOSA et al., 2014). O positivismo no Brasil passa a reforçar o autoritarismo doutrinário. Busca-se o progresso desde que a ordem prevaleça.

Ainda nessa época, a educação continuava voltada apenas para a classe dominante, a população de baixa- renda, na maioria negros e afrodescendentes, permanecia fora da escola ou recebia apenas uma educação profissionalizante (BARBOSA et al., 2014). Educação essa, excludente e que favorecia o poder político, econômico, e cultural da elite dominante. Nesse contexto, a saúde também não era considerada importante, o único interesse do estado era garantir o lucro da produção industrial.

Anos mais tarde, no século XX, especialmente na década de 1980, é evidente a segregação social que gera dificuldades de acesso à infraestrutura urbana e aos serviços essenciais à qualidade de vida à população, tais como: transporte, moradia, saneamento, saúde, educação (BARBOSA et al., 2014).

Embora o Ministério da Saúde apresenta elementos que pressupõem uma integração entre profissionais de saúde, gestores e população, ainda existe um distanciamento entre teoria e prática (BENITES FALKENBERG, et al., 2014). Dessa forma, entende-se que apesar das iniciativas do Estado em relação aos processos de cuidado de saúde da população, ainda existe um abismo entre o que se prega e o que de fato é realizado.

A relação entre saúde e educação tem se resumido a perguntas e respostas, em que as dificuldades diárias de um ambiente escolar são convertidas em diagnósticos individuais que com a ajuda da saúde poderiam ser resolvidos (SILVA, 2016). A saúde, por muito tempo, era vista de forma desvinculada às questões sociais, apenas como ausência de doença, sem fazer relação com os vários aspectos da vida humana.

A saúde deve ser compreendida de uma forma ampla que envolva os vários aspectos da vida de um indivíduo, ela "é um conceito positivo, que enfatiza os recursos sociais e pessoais, bem como as capacidades físicas" (BRASIL, 2002). Diante dessas concepções, com o passar dos anos, políticas públicas foram implantadas em relação à promoção da saúde em ambientes escolares.

\section{Aspectos relacionados à saúde no ambiente escolar}

A temática saúde e educação estão intimamente ligadas, e é inegável que o ambiente escolar trabalhe questões relacionadas à saúde (CARVALHO, 2015). A escola 
tem um forte papel de promoção à saúde a partir de práticas pedagógicas voltadas para essa temática, porém ainda enfrenta desafios em relação a esse trabalho, que muitas vezes precisa de parcerias intersetoriais, que depende de todo um conjunto de setores e ações para que funcione de forma significativa, garantindo o acesso à saúde, também nos ambientes escolares.

As ações educativas em saúde passaram a acontecer nos discursos oficiais a partir de 1889, época da primeira república, e suas ações eram voltadas apenas para os aspectos higienistas, que visavam um indivíduo sadio e produtivo (CARVALHO, 2015). Sendo assim, a saúde ainda não era percebida de uma forma mais ampla, mas apenas como a ausência de doença, conceito esse, que foi desconstruído ao longo do tempo.

$\mathrm{O}$ ambiente escolar pode promover estratégias a partir de práticas pedagógicas e de parcerias intersetoriais, que permitam que a promoção da saúde deixe de ser vista como apenas uma ação de "medicalização" (BARROS, 2002), ou seja, vista apenas como dependentes da oferta de serviços médicos, ou como ações pontuais e higienistas. A saúde deve ser compreendida de uma forma ampla que envolva os vários aspectos da vida de um indivíduo, ela "é um conceito positivo, que enfatiza os recursos sociais e pessoais, bem como as capacidades físicas" (BRASIL, 2002).

Atualmente a temática saúde na escola recebe atenção de diversos órgãos mundiais, em especial da Organização Mundial da Saúde (OMS) e da Organização das Nações Unidas para Educação, a Ciência e a Cultura (UNESCO).

Para as ações voltadas à saúde na escola, o Brasil instituiu em 2007, o Programa Saúde na Escola (PSE), que tem como objetivo trabalhar e atender aos princípios e diretrizes do Sistema Único de Saúde (SUS), que são: integralidade, equidade, universalidade, descentralização e participação social (CARVALHO, 2015). O PSE foca em ações voltadas à promoção da saúde no ambiente escolar com o intuito de promover uma formação ampla para a cidadania, com a participação não somente de escolar, mas também de seus familiares, e da comunidade como um todo.

Os projetos apoiados pela rede comunitária local revelam-se como iniciativas que oportunizam a problematização dos determinantes sociais do processo saúde-doença, favorecendo a construção de novas representações discursivas sobre a escola como espaço para a formação cidadã (PINTO, et al., 2020, p. 6).

Há a necessidade de se discutir nos ambientes escolares a relação existente entre a saúde e seus determinantes mais gerais, possibilitando a construção da aprendizagem 
permanente para os estudantes (TAVARES, 2006). Sendo assim, esse trabalho necessita da participação de outros espaços como: comunidade, família, serviços de saúde; por meio de ações simples e a participação de todos (CARVALHO, 2015).

Portanto, é importante que os setores da saúde e educação sejam trabalhados em conjunto, a partir da intersetorialidade, utilizando o espaço e as práticas pedagógicas escolares como meio de promoção à saúde, contribuindo assim, para a formação de um cidadão saudável, crítico e reflexivo.

\section{A importância da intersetorialidade na promoção da saúde dos estudantes}

No século XX a relação entre saúde e educação ainda era vista somente como um meio de trabalhar aspectos higienistas nas condutas individuais e comportamentais dos estudantes (PINTO, 2020). Com o passar do anos, entende-se que saúde envolve muitos outros aspectos, que vão além, do biológico, mas também, do social (LÓPEZFERNANDEZ, 2017; MALTA, et al., 2016).

Diante dessa perspectiva, políticas públicas voltadas para educação e saúde foram implantadas, como o PSE, com o objetivo de levar a promoção da saúde para os ambientes escolares, bem como para as comunidades onde estão inseridos os estudantes (CARVALHO, 2015). Proporcionando assim, um trabalho intersetorial na promoção da saúde e bem-estar dos estudantes.

A atuação interdisciplinar entre educação e saúde, "independentemente de onde ocorra - escola ou serviço de saúde - constitui um caminho importante para a conquista da qualidade de vida" (CARVALHO, 2015). Construir práticas pedagógicas relacionadas a esses dois importantes setores, é um grande desafio frente às demandas que as escolas enfrentam.

A escola é um importante espaço para as discussões e práticas que promovam a saúde dos escolares, bem como da comunidade em torno desse espaço.

A escola tem representado um importante local para o encontro entre saúde e educação abrigando amplas possibilidades de iniciativas tais como: ações de diagnóstico clínico e/ou social estratégias de triagem e/ou encaminhamento aos serviços de saúde especializados ou de atenção básica; atividades de educação em saúde e promoção da saúde (CASEMIRO, 2014, p. 830). 
No Brasil, nos últimos anos, surgiram políticas públicas voltadas para a promoção da saúde em ambientes escolares (PSE), e estudos voltados para esses campos, porém ainda existem desafios para esse trabalho:

Este desafio remete a três conceitos que podem e devem ser aprimorados e problematizados na perspectiva de atuação em saúde escolar, são eles: intersetorialidade, interdisciplinaridade e participação. Esta constatação não é uma novidade em termos de políticas de promoção da saúde (CASEMIRO, 2014, p. 836).

Trabalhar a intersetorialidade exige integração e coordenação entre setores, de maneira que se garanta a partilha dos desafios a serem enfrentados: orçamento, definição de estratégias e objetivos construídos em conjunto (BURLANDY, 2009).

Sendo assim, a efetivação da saúde escolar como política pública:

Requer a definição de iniciativas interdisciplinares selecionadas a partir de diagnóstico local da realidade, com identificação dos problemas reais e das soluções viáveis em cada escola de forma a contribuir para a autonomia e o apoderamento dos sujeitos diante dos direitos fundamentais relacionados ao tema da saúde escolar: direito à saúde, à educação, à alimentação e à vida digna (CASEMIRO, 2014, p. 839).

De acordo com Casemiro, “As definições necessárias para efetivar intersetorialidade, interdisciplinaridade e participação não ocorrem no vazio, mas contextualizadas pela realidade socioeconômica e política em nível micro e também macro" (CASEMIRO, 2014). Isto significa, que é importante compreender que as políticas públicas de saúde escolar precisam acontecer dentro de um contexto democrático e participativo, já que vivenciar o conceito ampliado de saúde depende do cenário em que essas ações ocorrem.

\section{Promoção da saúde e prevenção de agravos à saúde no ambiente escolar}

A promoção em saúde há anos tem sido discutida no cenário mundial e foi fortalecida na Conferência de Ottawa, nesta ratificou o conceito de saúde como qualidade de vida, condicionada a vários fatores como: renda, habitação, paz, educação, alimentação, ecossistema estável, recursos sustentáveis, justiça social e equidade (LÓPEZ-FERNANDEZ, 2017; MALTA, et al., 2016).

No Brasil observa-se o fortalecimento das ações de promoção à saúde na escola, a partir da articulação entre os setores de educação, saúde e sociedade. A comunidade escolar é a responsável no diagnóstico das necessidades, dos problemas de saúde e na 
definição de metodologias adequadas para abordar e enfrentar os problemas identificados (BRASIL, 2011).

Para o alcance dos objetivos do PSE é necessário que a intersetorialidade seja efetiva nos campos da saúde e educação nos territórios onde se encontram as escolas e as equipes de saúde da família (eSF). Essa sinergia pode garantir acesso a qualidade de uma vida melhor para crianças, adolescentes e aos jovens, porém alguns pontos devem ser considerados entre eles, podemos citar: 1) A atuação da eSF não deve competir ou sobrepor a atividade do professor ou atividade dos educandos; 2) No que diz respeito ao espaço físico a eSF deve considerar e respeitar as atividades já programadas pela escola; 3) As intervenções de saúde realizadas pela eSF (educativa, preventiva, clínica, etc) deve ter cunho pedagógico desde a intenção a execução; deve está em sintonia com a programação pedagógica da escola como também deverá ter a carga horária contada como momento de aprendizagem; 4) Os estudantes devem ser preparados sobre as atividades que serão desenvolvidas pela eSF e não somente avisados (BRASIL, 2011).

De acordo com a Política Nacional de Promoção à saúde, a promoção de saúde de acordo com a definição do SUS pode ser uma estratégia de articulação transversal utilizada pelo PSE, onde os educandos vão reconhecer os fatores que colocam a saúde da população em risco e às diferenças entre necessidades, territórios e culturas presentes no Brasil; com intuito que estes criem mecanismos que possam reduzir essas situações de vulnerabilidades. Segundo a OMS, a promoção da saúde objetiva ampliar as possibilidades da população de controlar os determinantes sociais da saúde e com isso melhorar a qualidade de vida (BRASIL, 2011).

Diante desta perspectiva, o PSE veio para apoiar na redução de agravos de saúde que provocavam o mau aproveitamento educacional de crianças e adolescentes da rede pública de educação do nosso país, podemos considerar como um grande marco pregado pelo SUS o da intersetorialidade e da corresponsabilização dos agentes envolvidos no processo (FARIAS, 2016).

Escolares bem informados serão adultos mais responsáveis e conscientes, poderão transmitir os conhecimentos adquiridos na escola em sua casa, família, vizinhos e amigos e, consequentemente disseminaram boas maneiras para uma melhoria nas condições de saúde mundial (MEDEIROS, 2011; VERDELONE, 2018). 


\section{CONSIDERAÇÕES FINAIS}

A partir desse estudo percebe-se que esses dois campos quando trabalhados de forma conjunta, refletem positivamente na qualidade de vida dos educandos, visto que, os indivíduos mais saudáveis aprendem melhor, e indivíduos com mais conhecimento são mais saudáveis.

Dessa forma é imprescindível que haja políticas públicas voltadas para o trabalho com esses dois setores. O Estado tem a obrigatoriedade de garantir a uma criança que ela seja saudável e que tenha acesso a uma educação de qualidade. Infelizmente, na história do Brasil Colonial, nota-se um descaso em relação a essa inter-relação, já que o único objetivo dos colonizadores era garantir lucros, sem se preocupar em oferecer aos habitantes da terra uma vida digna com acesso à saúde e educação. Esses aspectos só foram pensados a partir da chegada da família real, porém o acesso restrito para a elite.

Essas questões mostram que, no Brasil, não se costumava oferecer à população das classes baixas o acesso à práticas de promoção à saúde, bem como à educação. Práticas essas, que refletem na sociedade com a desigualdade social, onde poucos eram privilegiados e a maioria deixava de ter acesso a questões tão essenciais para o desenvolvimento físico e intelectual do indivíduo como: saúde e educação.

Percebe-se que mesmo com o passar dos tempos, nos séculos IX e XX, as questões voltadas para saúde e educação ainda não são percebidas e valorizadas de forma ampla, já que a única preocupação é capacitar um indivíduo para o mercado de trabalho. A saúde ainda era vista apenas como a ausência de doença, ou seja, desvinculada às questões sociais, sem fazer qualquer relação com os vários aspectos da vida humana.

Ao passar dos anos, ainda no século XX, os olhares passam a se modificarem em relação à interdependência entre saúde e educação. A escola passa a ser vista como um forte local para a promoção da saúde, deixando de lado apenas os aspectos higienistas e passando a se preocupar com o indivíduo como um ser social.

Devido aos grandes órgãos mundiais, como a OMS e a UNESCO, passarem a discutir a interdependências entre esses dois setores: saúde e educação, o Brasil cria, em 2007, o Programa Saúde na Escola (PSE) que estreita a relação entre esses dois campos, tentando garantir: a integralidade, equidade, universalidade, descentralização e participação social. Isso reflete de forma positiva para a sociedade, pois entende-se que esses direitos precisam ser garantidos a todos. 
Nesse contexto, a saúde não era mais vista apenas como forma de promover aspectos higienistas para o sujeito, mas sim, de garantir promoção da saúde no ambiente escolar com o intuito de promover uma formação ampla para a cidadania, com a participação não somente de escolar, mas também de seus familiares, e da comunidade como um todo. Situações essas, que refletem um grande avanço no Estado.

A partir dessa concepção, surge também a necessidade do diálogo com diversos setores: comunidade, família, serviços de saúde, escola; situações essas de intersetorialidade que fortalecem a promoção da saúde no ambiente escolar e promovem a formação de um sujeito crítico e reflexivo.

A intersetorialidade fortalece essa interdependência e permite que o trabalho em conjunto contribua para a efetividade das práticas pedagógicas voltadas para a promoção da saúde. Ela é um caminho importante para o fortalecimento da qualidade de vida de uma comunidade, mas apesar do seu valor, existem muitos desafios para esse trabalho.

O trabalho com a intersetorialidade exige um esforço coletivo de integração e coordenação entre os setores, de maneira que se garanta a partilha dos desafios para os setores envolvidos, que são as questões relacionadas a orçamento, definição de objetivos e estratégias elaboradas em conjunto.

Esse trabalho vai além da simples busca de apoio entre os setores, pois requer uma mudança de cultura, e de forma de organizar políticas públicas que garantam a divisão dos problemas apresentados ao longo do percurso. Isto significa, que é importante compreender que as políticas públicas de saúde escolar precisam acontecer dentro de um contexto democrático e participativo, já que vivenciar o conceito ampliado de saúde depende do cenário em que essas ações ocorrem.

Diante dessas proposições, entende-se que a inter-relação entre saúde e educação é de suma importância para o processo de desenvolvimento humano físico, emocional e social. E que apesar do Brasil ter avançado em relação a garantir um trabalho coletivo no que se refere, aos aspectos relacionados a esses dois campos, ainda existem muitos entraves que precisam ser vencidos, no qual a intersetorialidade seja trabalhada de forma a garantir entre os setores as responsabilidades inerentes a esse trabalho.

\section{REFERÊNCIAS}

BARROS, J. A. C. Pensando o processo saúde doença: a que responde o modelo biomédico? Saúde e Sociedade, v.11, n.1, p. 1-11, 2002. 
BARBOSA, A. S. et al. Reflexões sobre a saúde e a educação a partir de suas relações com o estado e a sociedade no Brasil. Revista Espaço para a Saúde, vol. 15, núm. 2, p. 05-20, 2014.

BENITES FALKENBERG, M.; LIMA MENDES, T. P.; PEDROZO DE MORAES, E.; DE SOUZA, E. M. Educação em saúde e educação na saúde: conceitos e implicações para a saúde coletiva. Ciência \& Saúde Coletiva, vol. 19, núm. 3, p. 847-852, 2014. https://doi.org/10.1590/1413-81232014193.01572013

BIRCH, D.A.; AULD, M.E. Public Health and School Health Education: Aligning Forces for Change. Health Promot Pract, v. 20, n.6, p. 818-823, 2019. https://doi:10.1177/1524839919870184.

BRASIL. As Cartas da Promoção da Saúde. Brasília: Ministério da Saúde, 2002.

BRASIL. Ministério da Saúde (BR). Departamento de Atenção Básica. Passo a passo PSE. Programa Saúde na Escola: tecendo caminhos da intersetorialidade [internet]. Brasília: Ministério da Saúde, 2011.

BURLANDY L. A construção da política de segurança alimentar e nutricional no Brasil: estratégias e desafios para a promoção da intersetorialidade no âmbito federal de governo. Cien Saude Colet, 14(3): 851-860, 2009. https://doi.org/10.1590/S141381232009000300020

CARVALHO, F. F. B. A saúde vai à escola: a promoção da saúde em práticas pedagógicas. Revista de Saúde Coletiva, Rio de Janeiro, 25 (4): p. 1207-1227, 2015. https://doi.org/10.1590/S0103-73312015000400009

CASEMIRO, J. P.; FONSECA, A. B. C.; SECCO, F. V. M. Promover Saúde na Escola: reflexões a partir de uma revisão sobre saúde escolar na América Latina. Ciência \& Saúde Coletiva. v. 19. n.3, p. 829-840, Rio de Janeiro, 2014. https://doi.org/10.1590/1413-81232014193.00442013

DOUGLAS, C., BIANCA, G. C. A percepção dos gestores escolares sobre a promoção de saúde nas escolas públicas. ECCOM, v.12, n.24, jul/dez.2021.

FARIAS, I.C.V., et al. Análise da intersetorialidade no programa saúde na escola. Revista Brasileira de Educação Médica, 40(2): 261-267, 2016.

LÓPEZ-FERNÁNDEZ, L.A, HORMAZÁBAL, O.S. Repensar la Carta de Ottawa 30 años después. Gac Sanit, 31(6):443-5, 2017. https://doi: 10.1016/j.gaceta.2016.12.013

LOPES, I.E., et al. Eixos de ação do programa saúde na escola e promoção da saúde: revisão integrativa. Saúde Debate,42(128): 773-789, 2018.

MALTA, C.D. et al. National Health Promotion Policy (PNPS): chapters of a journeystill under construction. Ciênc Saúde Colet., 21(6):1683-94, 2016. https://doi: 10.1590/1413-81232015216.07572016

MEDEIROS, A. B. et al. A importância da educação Ambiental na escola nas series iniciais. Revista Faculdade Montes Belos, v.4, n.1, set. 2011. 
PINTO, M. B.; SILVA, K. L. Promoção da saúde na escola: discursos, representações e abordagens. Rev., Bras. Enferm., Brasília, v.73, n.3, e20180774, 2020.

https://doi.org/10.1590/0034-7167-2018-0774

SILVA, C. C. B.; MOLERO, E. S. S.; ROMAN, M. D. A interface entre saúde e educação: percepção de educadores sobre educação inclusiva. Psicologia Escolar e Educacional, vol. 20, num. 1, p. 109-115, jan/abr 2016. https://doi.org/10.1590/2175353920150201934

TAVARES, M. F. L.; ROCHA, R. M. Promoção da Saúde e a Prática de Atividade Física em Escolas de Manguinhos - Rio de Janeiro. BRASIL. Ministério da Saúde. Escolas promotoras de saúde: experiências do Brasil. Brasília: Ministério da Saúde, Organização Pan-Americana da Saúde, 2006.

UPE. Campus Petrolina - Pós-graduação e pesquisa: Stricto sensu - PPGFPPI o Programa. Disponível em: http://w2.portais.atrio.scire.net.br/upe-ppgfppi/. Acesso em: 06 set. 2021.

VERDELONE, T. H.; CAMPBELL, G.; ALEXANDRINO, C. R. Trabalhando educação ambiental com turmas do ensino fundamental I. Brazilian Journal of Development, v.5, n.6, p.4675-4687, 2018.

\section{Recebido em: 05/11/2021}

Aprovado em: 02/12/2021

Publicado em: 08/12/2021 